\title{
Identification of the different magnetic field contributions during a geomagnetic storm in magnetospheric and ground observations
}

\author{
Tommaso Alberti ${ }^{1}$, Mirko Piersanti ${ }^{2}$, Antonio Vecchio ${ }^{3}$, Paola De Michelis ${ }^{4}$, Fabio Lepreti ${ }^{1}$, Vincenzo Carbone ${ }^{1}$, and \\ Leonardo Primavera ${ }^{1}$ \\ ${ }^{1}$ Department of Physics, University of Calabria, Rende (CS), Italy \\ ${ }^{2}$ Department of Physical and Chemical Sciences, University of L'Aquila, Italy \\ ${ }^{3}$ LESIA-Observatoire de Paris, 5 place Jules Janssen, 92190 Meudon, France \\ ${ }^{4}$ Section of Geomagnetism, Aeronomy and Enviromental Geophysics, Istituto Nazionale di Geofisica e \\ Vulcanologia, Roma, Italy
}

Correspondence to: Tommaso Alberti (tommaso.alberti@unical.it)

Received: 13 June 2016 - Revised: 27 October 2016 - Accepted: 28 October 2016 - Published: 22 November 2016

\begin{abstract}
We used the empirical mode decomposition (EMD) to investigate the time variation of the magnetospheric and ground-based observations of the Earth's magnetic field during both quiet and disturbed periods. We found two timescale variations in magnetospheric data which are associated with different magnetospheric current systems and the characteristic diurnal orbital variation, respectively. On the ground we identified three timescale variations related to the solar-wind-magnetosphere high-frequency interactions, the ionospheric processes, and the internal dynamics of the magnetosphere. This approach is able to identify the different physical processes involved in solar-windmagnetosphere-ionosphere coupling. In addition, the largetimescale contribution can be used as a local index for the identification of the intensity of a geomagnetic storm on the ground.
\end{abstract}

Keywords. Magnetospheric physics (magnetosphereionosphere interactions; solar-wind-magnetosphere interactions; storms and substorms)

\section{Introduction}

Geomagnetic storms (GSs) are global magnetic disturbances that result from the interaction between magnetized plasma propagating from the Sun and magnetic fields in the nearEarth space environment. They are often accompanied by strong perturbations of the geomagnetic field that can generate intense disturbances of the terrestrial environments and affect experimental devices and communications (i.e., high-frequency communications, GNSS navigation, and so on) (Goodman, 2005; Schwenn, 2006; Pulkkinen, 2007; Gonzáles-Hernández et al., 2014). The forecasting of these events (space weather), their geoeffectiveness, and the associated GSs are then of primary interest.

GS strength is assessed by the disturbance storm time (Dst) index that reveals the decrease in the horizontal geomagnetic field component and gives a global measure of midlatitude magnetic disturbance. The Dst index has long been used as an indirect measure of the ring current that encircles the Earth at altitudes ranging from about 3 to 7 Earth radii $\left(R_{\mathrm{E}}\right)$ and increases its intensity during GSs. Indeed, Dst values are proportional to the total kinetic energy of the particles that form the ring current according to the DesslerParker-Sckopke relationship (Dessler and Parker, 1959; Sckopke, 1966). Nowadays, it is known that this index measures the effects of many terrestrial and magnetospheric current systems which are enhanced during geomagnetic disturbed periods. Dst is an hourly index (measured in nanotesla) obtained by finding the instantaneous average of the deviations from a quiet day in the horizontal component of the magnetic field at four observatories that are sufficiently distant from the auroral and equatorial electrojets and distributed in longitude as evenly as possible (McPherron et al., 1986). It has been recently suggested that local geomagnetic indices could represent very useful tools, complementary to global indices, in defining deviations from the usual geomagnetic activity. In fact, local geomagnetic disturbances seem to play 
a key role in assessing the potential risk factor of extreme events in specific regions (Cid et al., 2014). So, it can be useful to investigate the time evolution of a baseline of the geomagnetic field defined as the local geomagnetic activity observed on large scales.

From a theoretical point of view, the separation between a time-dependent background magnetic field (baseline) and its fluctuations does make sense under the implicit assumption of a clear timescale separation between the fluctuations and the background. The validity of this assumption is questionable due to the nonlinear response of the geomagnetic system to the complex external forcing provided by the solar wind (SW). It is clear that neither the scale separation nor the stationarity of the signal are assured when the geomagnetic field is observed. When a timescale separation is not present, it is difficult to identify an average magnetic field.

In the present paper, using the empirical mode decomposition (EMD), we suggest a method to separate the average magnetic field by its variations, identifying the physical meaning of each contribution.

\subsection{Empirical mode decomposition}

The EMD has been developed to process nonstationary data (Huang et al., 1998) and successfully applied in many different contexts (Cummings et al., 2004; McDonalds et al., 2007; Terradas et al., 2004; Vecchio et al., 2010; Wu and Huang, 2009), including geophysical systems (Alberti et al., 2014; Balasis and Egbert, 2006; De Michelis et al., 2012; Jánosi and Müller, 2005; Wu et al., 2008; Zhen-Shan and Xian, 2007). It is an adaptive and a posteriori decomposition method in which the basis functions are derived from the data. This technique decomposes a set of observed data $X(t)$ into a finite number $m$ of intrinsic oscillatory functions $C_{j}(t)$, named intrinsic mode functions (MFs), so that

$X(t)=\sum_{j=1}^{m} C_{j}(t)+r(t)$,

where $r(t)$ is the final residue of the decomposition from which no more MFs can be extracted. Each mode $C_{j}(t)$ can be derived by the so-called sifting process, which represents the core of the decomposition procedure. This procedure can be summarized by the following steps:

1. identification of the local extrema of the time series $X(t)$

2. interpolation of local minima (maxima) by using a spline function to obtain the local envelope $e_{\min }(t)\left(e_{\max }(t)\right)$

3. computation of the average envelope $m_{1}(t)=$ mean $\left(\left[e_{\min }(t), e_{\max }(t)\right]\right)$

4. evaluation of the detail $h_{1}(t)=X(t)-m_{1}(t)$.
The previous steps are iterated $k$ times until the obtained detail $h_{1 k}=h_{1(k-1)}-m_{1 k}$ can be identified as an intrinsic MF that must satisfy the following two properties:

1. The number of extrema and the number of zerocrossings must either be equal or differ at most by 1 .

2. At any point (locally), the mean value of the envelope defined by the local maxima and by the local minima is 0 .

In addition, the number of sifting steps to produce an MF is defined by the stopping criterion proposed by Huang et al. (1998), similar to the Cauchy convergence test, which defines a sum of the difference (standard deviation), $\sigma_{k}$, between two sifting steps as

$\sigma_{k}=\sum_{t=0}^{T} \frac{\left|h_{k-1}(t)-h_{k}(t)\right|^{2}}{h_{k-1}^{2}(t)}$.

The sifting process stops when $\sigma_{k}$ is smaller than a given value, typically in the range $0.2-0.3$ (in the present work we used 0.3) (Huang et al., 1998; Alberti et al., 2014)

An MF is an oscillating function modulated in both amplitude and frequency, as $C_{j}(t)=A_{j}(t) \cos \left[\Phi_{j}(t)\right]$, where $\Phi_{j}(t)$ is the instantaneous phase of the $j$ th mode, related to the instantaneous frequency $\omega_{j}(t)=\mathrm{d} \Phi_{j}(t) / \mathrm{d} t$ (Huang et al., 1998). Since other decomposition techniques do not consider a time-dependent frequency (e.g., Fourier analysis), this concept of instantaneous frequency is the main point of the EMD technique, allowing a decomposition of nonstationary time series without any assumption on the basis of the decomposition. It can be derived by using the so-called HilbertHuang transform (Huang et al., 1998), through which for each $C_{j}(t)$ we can derive the corresponding Hilbert transform $\widetilde{C}_{j}(t)$ as

$\widetilde{C}_{j}(t)=\frac{1}{\pi} \mathbf{P} \int_{-\infty}^{+\infty} \frac{C_{j}\left(t^{\prime}\right)}{t-t^{\prime}} \mathrm{d} t^{\prime}$,

where $\mathbf{P}$ denotes Cauchy's principal value. The function defined by Eq. (3) exists for all $\mathbf{L}^{p}$ space functions, allowing us to define an analytical signal $Z(t)$ from the conjugate pair $\left(C_{j}(t), \widetilde{C}_{j}(t)\right)$, such that

$Z_{j}(t)=C_{j}(t)+i \widetilde{C}_{j}(t)=A_{j}(t) e^{i \Phi_{j}(t)}$,

in which $A_{j}(t)$ and $\Phi_{j}(t)$ are the instantaneous amplitude and phase of the $j$ th mode, respectively, derived as

$$
\begin{aligned}
A_{j}(t) & =\sqrt{C_{j}(t)^{2}+\widetilde{C}_{j}(t)^{2}}, \\
\Phi_{j}(t) & =\arctan \frac{\widetilde{C}_{j}(t)}{C_{j}(t)} .
\end{aligned}
$$


In this way, the instantaneous frequency can be derived by the instantaneous phase as $\omega_{j}(t)=\mathrm{d} \Phi_{j}(t) / \mathrm{d} t$ (Huang et al., 1998). Consequently, a typical average period $T_{j}$ can be estimated for all the MFs as $T_{j}=2 \pi /<\omega_{j}(t)>_{\mathrm{t}}\left(<>_{\mathrm{t}}\right.$ representing the time average). The decomposition is clearly local and complete, which means that the MFs can reconstruct the original signal (see Eq. 1), while the orthogonality property is not theoretically ensured. However, it can be verified by evaluating the orthogonal index (OI) as proposed in Huang et al. (1998) by checking the inner product of each MF with respect to the others. In this case, the EMD can be used as a filter through partial sums of a subset of modes (Alberti et al., 2014). Finally, the statistical significance of each MF with respect to white noise can be verified through the test developed by Wu and Huang (2004), which allows a comparison of the mean square amplitude of the MFs with the theoretical spread function of white noise computed for different confidence levels.

In addition, EMD is capable of overcoming some limitations of other decomposition analysis techniques. EMD does not require any a priori assumption on the functional form of the basis of the decomposition (as for Fourier or wavelet analysis). In this way, several misleading results can be avoided, and this allows us to carry out local nonstationary and nonlinearity features from each time series, which are usually far from the decomposition properties obtained with fixed eigenfunctions. However, as for other analysis techniques we need to outline outstanding open problems with EMD, including end effects of the EMD or stopping criteria selection. More specifically, boundary effects occur because there is no point before the first data point and after the last data point. In most cases, these boundary points are not the extreme value of the signal; therefore, they can cause the divergence of the extreme envelope, causing significant errors. These errors can produce misleading MF waveforms at its endpoints, which can propagate into the decomposition through the sifting process. To avoid problems due to boundary effects, various methods have been proposed, including mirror- or data-extending methods (Huang and $\mathrm{Wu}, 2008$; Yang et al., 2014). In our case, we used the data-extending method by adding a constant extension to the boundary points of the maxima and minima vectors, allowing to get a better spline fit at the ends.

\subsection{Standardized mean test (SMT)}

In order to study the physical meaning of the EMD modes and connect them to the timescales involved in the variations of magnetospheric and geomagnetic field, we used the method proposed by Flandrin et al. (2004). They suggest that, if a clear timescale separation exists in a data set, this can be divided into two different contributions:

$X(t)=\delta X(t)+X_{0}(t)$, where $X_{0}(t)$ is the so-called baseline field and $\delta X(t)$ are variations around $X_{0}(t)$.

The basic idea is that $\delta X(t)$

- has a close to zero standardized mean (SM) (defined as the mean divided by the standard deviation)

- represents the fluctuating/oscillatory high-frequency contribution to the time series.

Using the orthogonality and completeness properties of EMD, we define $\delta X(t)$ as the reconstruction of a subset $S_{1}$ of $k<m$ empirical modes, which satisfies the previous two properties:

$\delta X(t)=\sum_{j=1}^{k} C_{j}(t)$.

The $k$ value represents the last mode index for which the reconstruction given by Eq. (8) has an SM close to 0. To investigate if the $k$ value could be dependent on the length of the considered time series, we applied the following procedure. First of all, for each $j$, we evaluate the SM on the whole time range considered. Then, we consider a set of $N_{\mathrm{S}}=1000$ time windows, with different lengths, moving within the whole time range; we evaluate the standardized mean for each partial reconstruction, obtaining a set of $N_{\mathrm{S}}$ values, and, consequently, we calculate the corresponding standard deviations at each $j$. In this way, we associate an error with each standardized mean evaluated over the whole time range, corresponding to 3 times the standard deviation for the considered partial reconstruction. For all the considered cases, the $k$ value remains unchanged within the error band, indicating that the SMT is not significantly dependent on the choice of the time window length. This implies that the first $k$ modes are able to reproduce the fluctuating contribution to the time series, while the remaining $m-k$ modes represent the larger timescale variations. This could be very useful for research into the framework of turbulent-like processes where a high-frequency component needs to be properly filtered out. Moreover, as shown in the next sections, the $k$ th mode characteristic timescale is also not dependent on the geomagnetic activity of the considered period, indicating that this separation between high- and low-frequency processes exists in both quiet and disturbed periods.

\section{Magnetospheric and ground observations: EMD approach}

In the present paper we show the results obtained when this approach is applied to a super solar quiet (SSQ) period and to a storm time (ST) event. In order to distinguish between SSQ and ST, we use the $K$-index. It quantifies disturbances in the horizontal $(H)$ and eastward $(D)$ components of the Earth's 
AQU: $\lambda_{\mathrm{g}}=42 . \mathrm{I0}^{\mathrm{O}} \mathrm{N} ; \phi_{\mathrm{g}}=13.32^{\circ} \mathrm{E}$
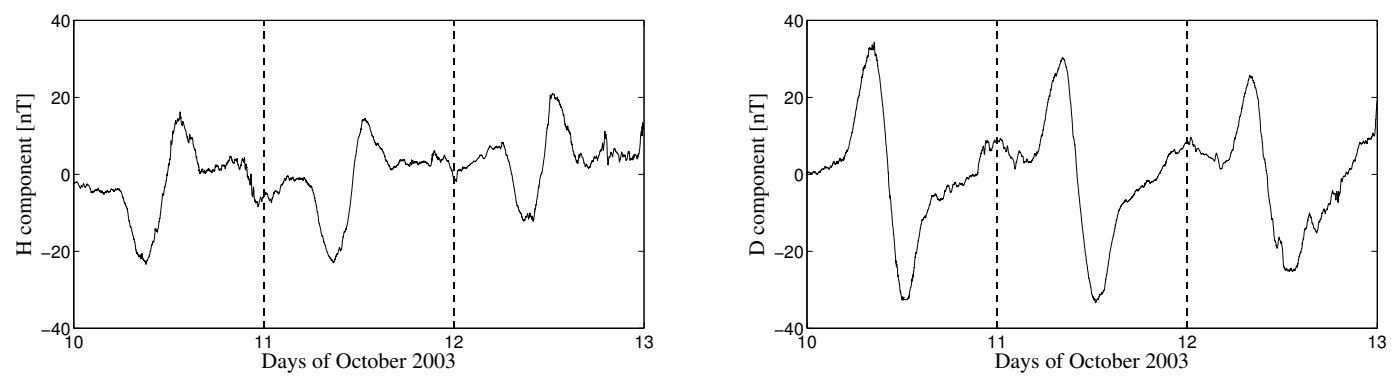

Figure 1. SSQ, 10-12 October 2003: midlatitude ground observations (AQU: $\lambda_{\mathrm{g}}=42.38^{\circ} \mathrm{N}$ and $\phi_{\mathrm{g}}=13.32^{\circ} \mathrm{E}$ ) for the $H$ (left panel) and $D$ (right panel) components. The time resolution is $1 \mathrm{~min}$.

magnetic field with an integer in the range of 0-9 with 1 being quiet and 5 or more indicating a geomagnetic storm. It is derived from the maximum fluctuations of $H$ or $D$ components observed on a magnetometer during a $3 \mathrm{~h}$ interval. According to this scheme, we define an SSQ period when $K<1$, while an ST event is identified when $K>5$. We consider, as ST event, the Halloween super storm that occurred between 28 October and 1 November 2003.

For our analysis, we used $1 \mathrm{~min}$ data from the horizontal component of the geomagnetic field $(H)$ and the eastward component $(D)$ measured at L'Aquila (AQU: $\lambda_{\mathrm{g}}=42.38^{\circ} \mathrm{N}$ and $\phi_{\mathrm{g}}=13.32^{\circ} \mathrm{E} ; \lambda_{\mathrm{g}}$ and $\phi_{\mathrm{g}}$ are the geographic latitude and longitude, respectively) permanent geomagnetic observatory to study the time variations of the geomagnetic field.

Moreover, we used 1 min geosynchronous satellite observations at $6.6 R_{\mathrm{E}}$ from Geostationary Operational Environmental Satellites (GOES) (GOES10: LT = UT-9; GOES12: $\mathrm{LT}=\mathrm{UT}-5$; both in the Geocentric Solar Ecliptic - GSE - coordinate system) for the magnetospheric field (http: //cdaweb.gsfc.nasa.gov/). More specifically, we used the

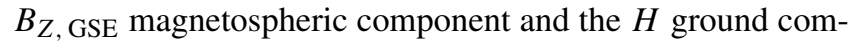
ponent. At high and middle latitudes, their behavior should be comparable because of the $90^{\circ}$ rotation of the polarization axes through the ionosphere (Hughes and Southwood, 1974; Sciffer et al., 2005; Piersanti et al., 2012; Waters et al., 2013).

\subsection{Super solar quiet period: 10-12 October 2003}

Figure 1 shows the observations of the geomagnetic field at AQU for the horizontal component of the vector along the local magnetic meridian ( $H$, left panel) and the eastward component $(D$, right panel) during the super solar quiet period 10-12 October 2003. During the entire period of interest, the $K$-index ranges between 0 and 1 ( $K$ being the $3 \mathrm{~h}$ long quasilogarithmic local index of geomagnetic activity for this observatory: http://roma2.rm.ingv.it/en/). Both $H$ and $D$ components show the typical midlatitude solar quiet $(\mathrm{Sq})$ daily variation (Chapman, 1929; Matsushida and Maeda, 1965; Chulliat et al., 2005; De Michelis et al., 2010). Sq is due to two different sources: the current systems flowing in the socalled ionospheric dynamo region and the induced telluric currents in the Earth's upper mantle. These currents, in turn, generate additional magnetic field variations that are almost in phase with the primary variations. The dynamo currents flowing in the E region of the Earth's ionosphere are driven by the global thermotidal wind systems and are dependent upon the local tensor conductivity and the main geomagnetic field vector. The morphology of the atmospheric tides gives the ionospheric currents a configuration characterized by two pairs of vortices: two great vortices in the sunlit hemisphere and the other two in the dark one. The two vortices in the sunlit hemisphere are the most intense because the ionospheric conductivity is larger. The pattern of the two current vortices in the sunlit hemisphere consists of currents circulating about foci at +30 and $-30^{\circ}$ magnetic latitude. Viewed from the Sun, circulation is counterclockwise in the Northern Hemisphere and clockwise in the Southern Hemisphere (Richmond et al., 1976; Takeda, 2002; Hawary et al., 2012; Yamazi and Yumoto, 2004; Shinbori et al., 2014). Applying the EMD procedure we found a set of 13 modes and 11 modes for the $H$ and $D$ component, respectively (see Fig. 2), while characteristic timescales are reported in Table 1.

Figure 3 shows the SMT applied to the EMD of the $H$ (left panel) and the $D$ (right panel) components. We found three different sets of modes: the modes $j=1-8$ for $H$ and $j=1-7$ for $D$ are identified as short-timescale reconstructions whose timescale is $\leq 4 \mathrm{~h}$ and for which the SM of the reconstructions is close to 0 ; the modes $j=9-12$ for $H$ and $j=8-10$ for $D$ are identified as intermediate-timescale reconstructions with a timescale in the range of 6-24h. The mode $j=13$ and the residue for $H$ and the mode $j=10$ and residue for $D$ are identified as large-timescale reconstructions for which the SM of the reconstructions departs significantly from 0 . For the intermediate timescales, a significant non-null value is observed when $j=9$ for $H(j=8$ for $D)$, while when $j=12(j=10$ for $D)$ an $\mathrm{SM} \sim 0$ is again obtained. The physical meaning of the intermediate modes will be discussed below. Moreover, we note the ex- 

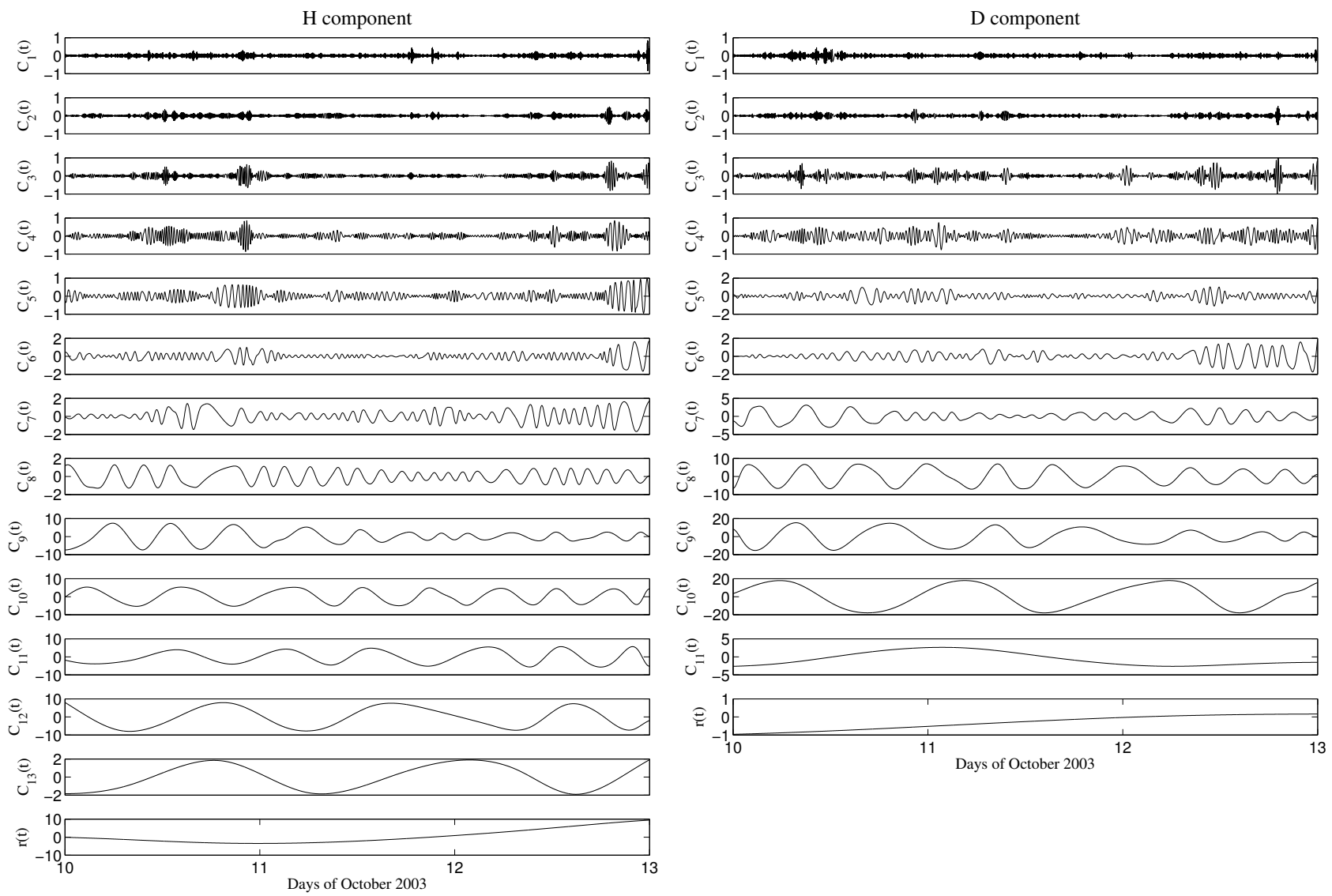

Figure 2. SSQ, 10-12 October 2003: empirical modes extracted via EMD from AQU $H$ component (left panels) and AQU $D$ component (right panels). The modes and the residue are expressed in nanotesla, and the time resolution is $1 \mathrm{~min}$.

Table 1. Mean periods of intrinsic modes during the super solar quiet period (the dash corresponds to the period of the residue $r(t)$ which cannot be evaluated). G10 and G12 refer to GOES10 and GOES12 satellites, respectively.

\begin{tabular}{rrrrr}
\hline \multicolumn{5}{c}{ Super solar quiet periods (h) } \\
\hline & $T_{H}$ & $T_{D}$ & $T_{G 10}$ & $T_{G 12}$ \\
\hline & $0.05 \pm 0.01$ & $0.05 \pm 0.01$ & $0.05 \pm 0.02$ & $0.05 \pm 0.01$ \\
& $0.10 \pm 0.02$ & $0.10 \pm 0.02$ & $0.16 \pm 0.03$ & $0.17 \pm 0.02$ \\
& $0.16 \pm 0.02$ & $0.19 \pm 0.02$ & $0.28 \pm 0.05$ & $0.29 \pm 0.04$ \\
$\delta H(D) / \delta B_{z}$ & $0.28 \pm 0.04$ & $0.40 \pm 0.03$ & $0.46 \pm 0.05$ & $0.51 \pm 0.06$ \\
& $0.51 \pm 0.04$ & $0.73 \pm 0.06$ & $2.20 \pm 0.4$ & $1.90 \pm 0.4$ \\
& $0.92 \pm 0.08$ & $1.4 \pm 0.2$ & $3.80 \pm 0.5$ & $3.0 \pm 0.5$ \\
& $1.7 \pm 0.2$ & $3.3 \pm 0.3$ & & $3.7 \pm 0.6$ \\
& $3.0 \pm 0.4$ & & & \\
\hline \multirow{5}{*}{$H^{*}\left(D^{*}\right)$} & $6.1 \pm 0.6$ & $8.2 \pm 0.6$ & & \\
& $8.7 \pm 0.7$ & $12 \pm 1$ & & \\
& $12 \pm 1$ & $24 \pm 1$ & & \\
$H_{0}\left(D_{0}\right) / B_{z 0}$ & $24 \pm 1$ & & & \\
& $34 \pm 2$ & $31 \pm 2$ & $24 \pm 1$ & $24 \pm 1$ \\
& - & - & $30 \pm 2$ & $32 \pm 3$ \\
& \multicolumn{5}{c}{$34 \pm 3$} & - \\
\hline
\end{tabular}


SMT results

[AQU: $\left.\lambda_{g}=42.38^{\circ} \mathrm{N} ; \phi_{g}=13.32^{\circ} \mathrm{E}\right]$
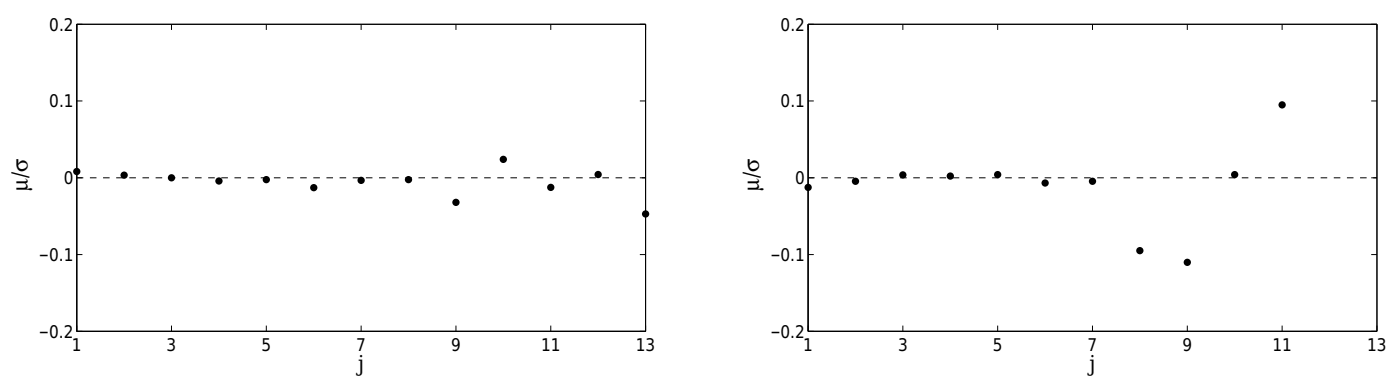

Figure 3. SSQ, 10-12 October 2003: SMT applied to the EMD reconstruction of the ground observations for the $H$ (left panel) and $D$ (right panel) components. The $x$ axis is order in terms of the last mode involved in the partial sums (see Eq. 8).
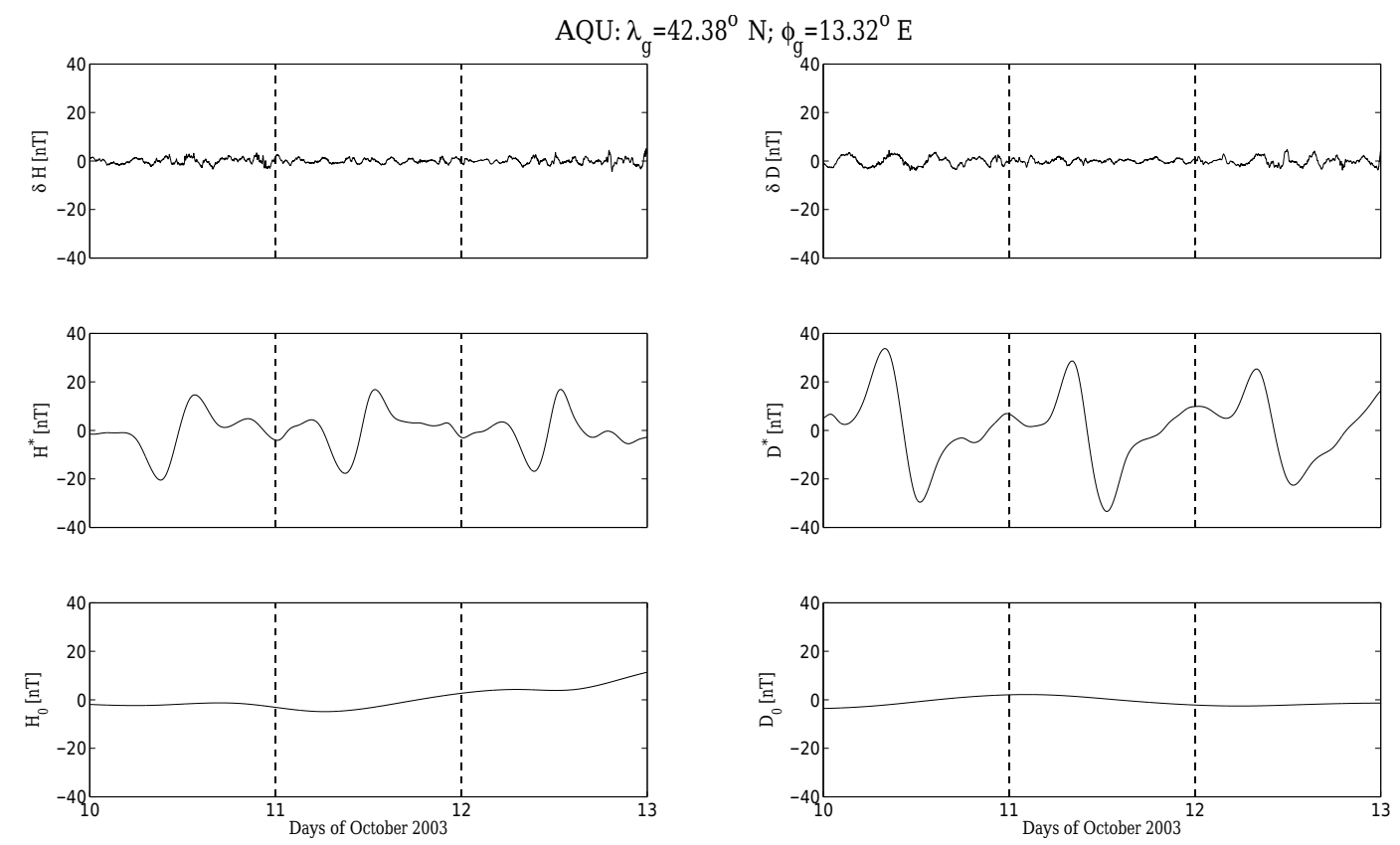

Figure 4. SSQ, 10-12 October 2003: EMD reconstruction for the $H$ (left panels) and $D$ (right panels) components at midlatitude ground station (AQU: $\lambda_{\mathrm{g}}=42.38^{\circ} \mathrm{N}$ and $\phi_{\mathrm{g}}=13.32^{\circ} \mathrm{E}$ ). The time resolution is $1 \mathrm{~min}$.

istence of short-timescale modes which are characterized by a low mean amplitude ( $j=1-8$ for $H, j=1-7$ for $D$ ), while modes with timescales greater than $\sim 4 \mathrm{~h}$ are characterized by higher mean amplitudes. In particular, $j=12$ for $H$ and $j=10$ for $D$ show the characteristic diurnal contribution in the geomagnetic components. According to the SMT results (Fig. 3), AQU data are split into three different contributions:

$H(t)=\delta H(t)+H^{*}(t)+H_{0}(t)$,

$D(t)=\delta D(t)+D^{*}(t)+D_{0}(t)$.

Figure 4 shows the EMD reconstructions for the $H$ (left panels) and $D$ (right panels) components. $\delta H(t)$ and $\delta D(t)$ (Fig. 4, upper panels) represent short-timescale reconstructions; $H^{*}(t)$ and $D^{*}(t)$ (Fig. 4, middle panels) are intermediate-timescale reconstructions; $H_{0}(t)$ and $D_{0}(t)$ (Fig. 4, lower panels) represent the large-timescale reconstructions.

In order to detect if the same timescale separation is found in the magnetosphere, we analyzed magnetospheric observations at a geosynchronous orbit by applying the same techniques. Figure 5 shows the time series obtained from GOES10 (left panel) and GOES12 (right panel) observations with a corresponding color scale related to the magnetic local time (MLT) of the spacecraft. Both GOES spacecraft show the characteristic orbital variation (see color scale related to the MLT). 

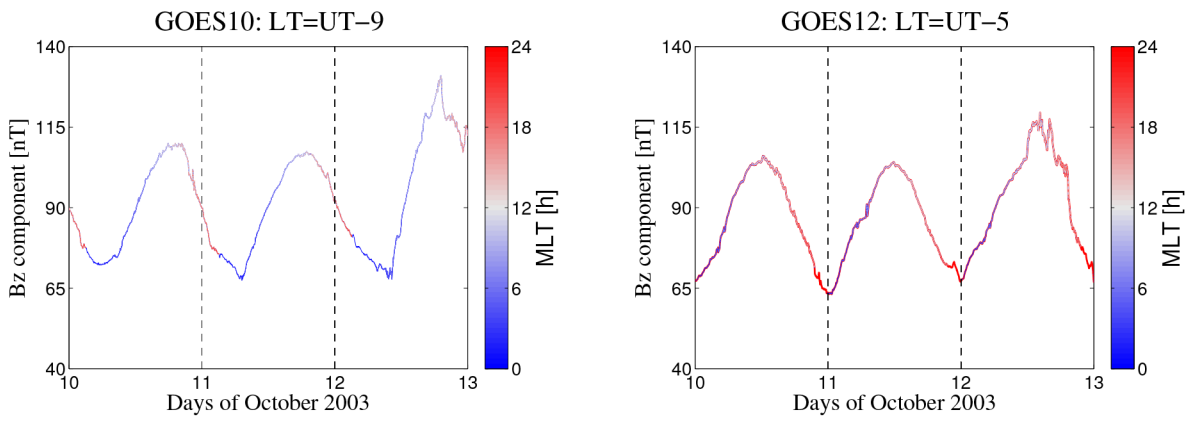

Figure 5. SSQ, 10-12 October 2003: magnetospheric observations at geosynchronous orbit for GOES10 (LT=UT-9; left panel) and GOES12 (LT $=\mathrm{UT}-5$; right panel) spacecraft. The time resolution is $1 \mathrm{~min}$.

GOES 10 Bz component
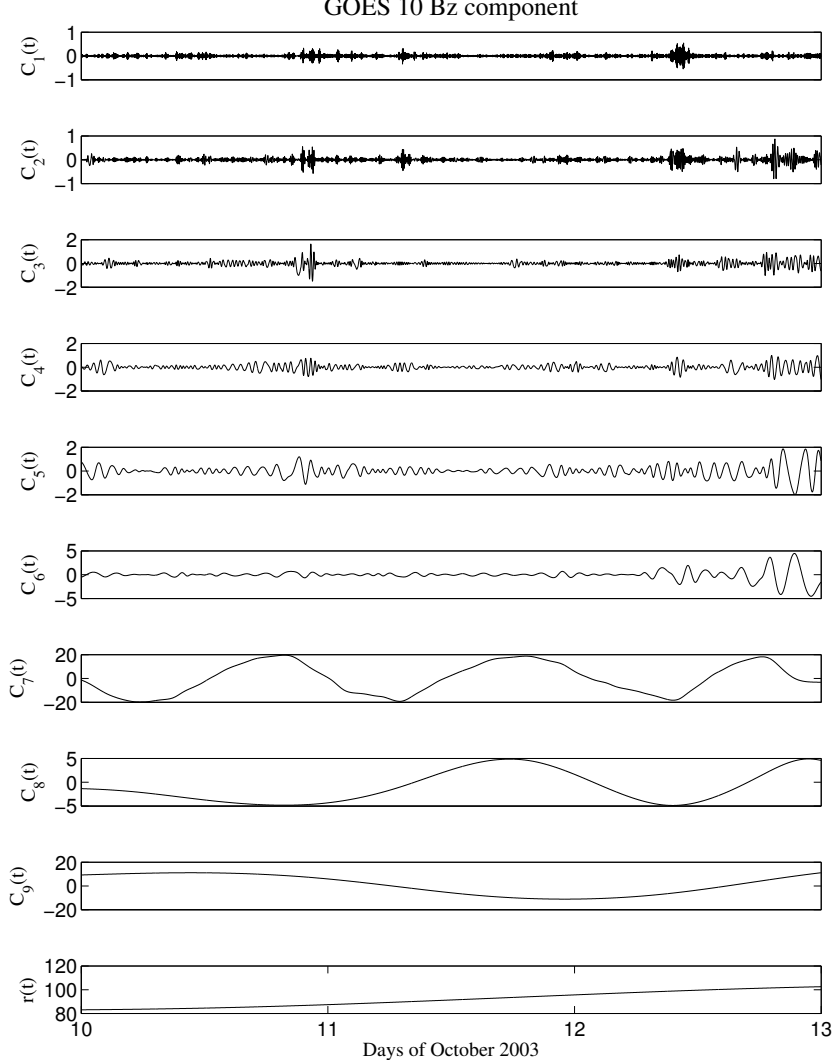

GOES 12 Bz component
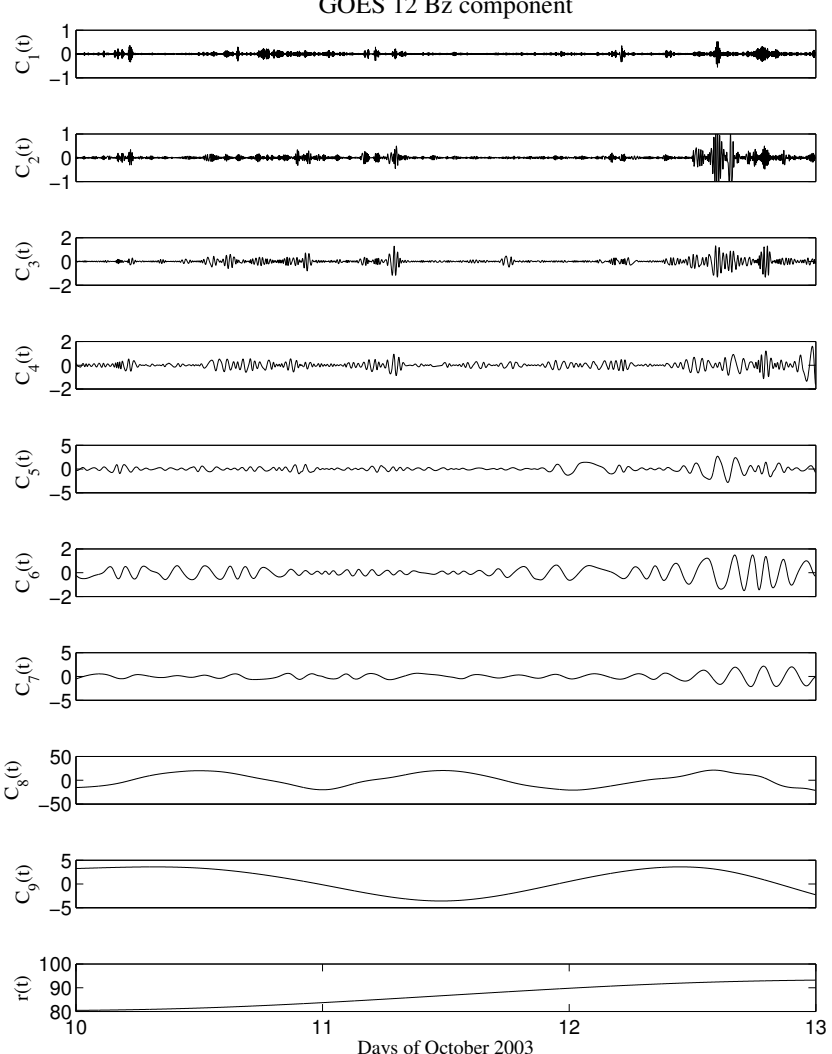

Figure 6. SSQ, 10-12 October 2003: empirical modes extracted via EMD from GOES10 (left panels) and GOES12 (right panels) $B_{z}$ components. The modes and the residues are expressed in nanotesla, with a time resolution of $1 \mathrm{~min}$.

Applying the EMD procedure, we obtained a set of nine modes for both GOES spacecraft, as shown in Fig. 6. We note that the modes $j=7$ for GOES10 and $j=8$ for GOES12 reproduce the diurnal orbital variation observed by the spacecraft. Moreover, the SMT results (see Fig. 7) reveal the existence of two timescales of variability, characterized by different mean amplitudes. For these reasons, both GOES10 (left panels) and GOES12 (right panels) are split into only two different sets of modes: the short-timescale reconstructions, characterized by a nearly zero mean contribution to the signal, and the large-timescale reconstructions, characterized by a non-zero mean contribution to the signal. So, according to the SMT results, GOES10 and GOES12 EMD results are split into two different contributions (see Fig. 8):

$B_{z}(t)=\delta B_{z}(t)+B_{z_{0}}(t)$,

where $\delta B_{z}(t)$ represents short-timescale reconstructions (GOES10: $j=1-6$ modes; GOES12: $j=1-7$ modes) and $B_{z 0}(t)$ represents the large-timescale reconstructions 


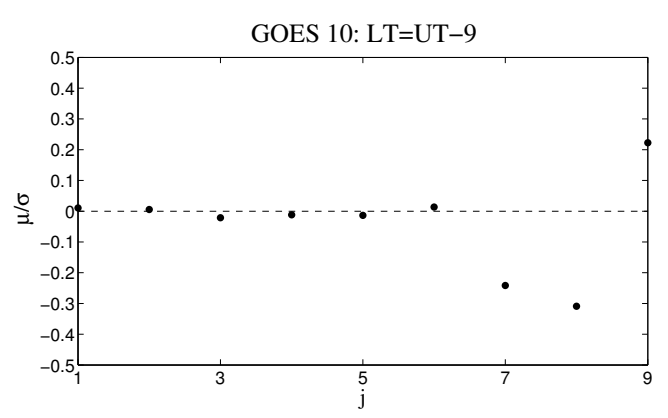

SMT results

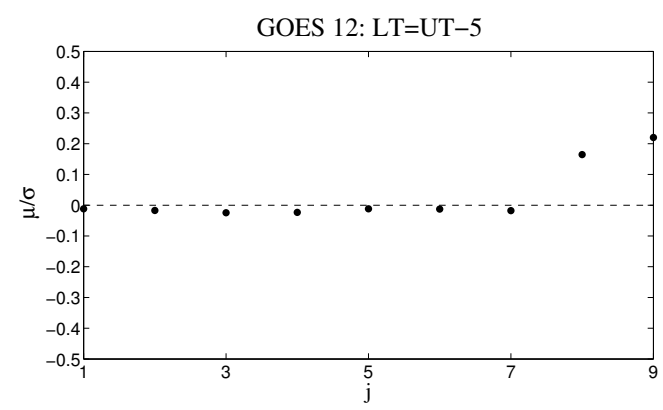

Figure 7. SSQ, 10-12 October 2003: SMT applied to the EMD reconstruction of the magnetospheric observations for GOES10 (left panel) and GOES12 (right panel) $B_{z}$ components. The $x$ axis is order in terms of the last mode involved in the partial sums (see Eq. 8).
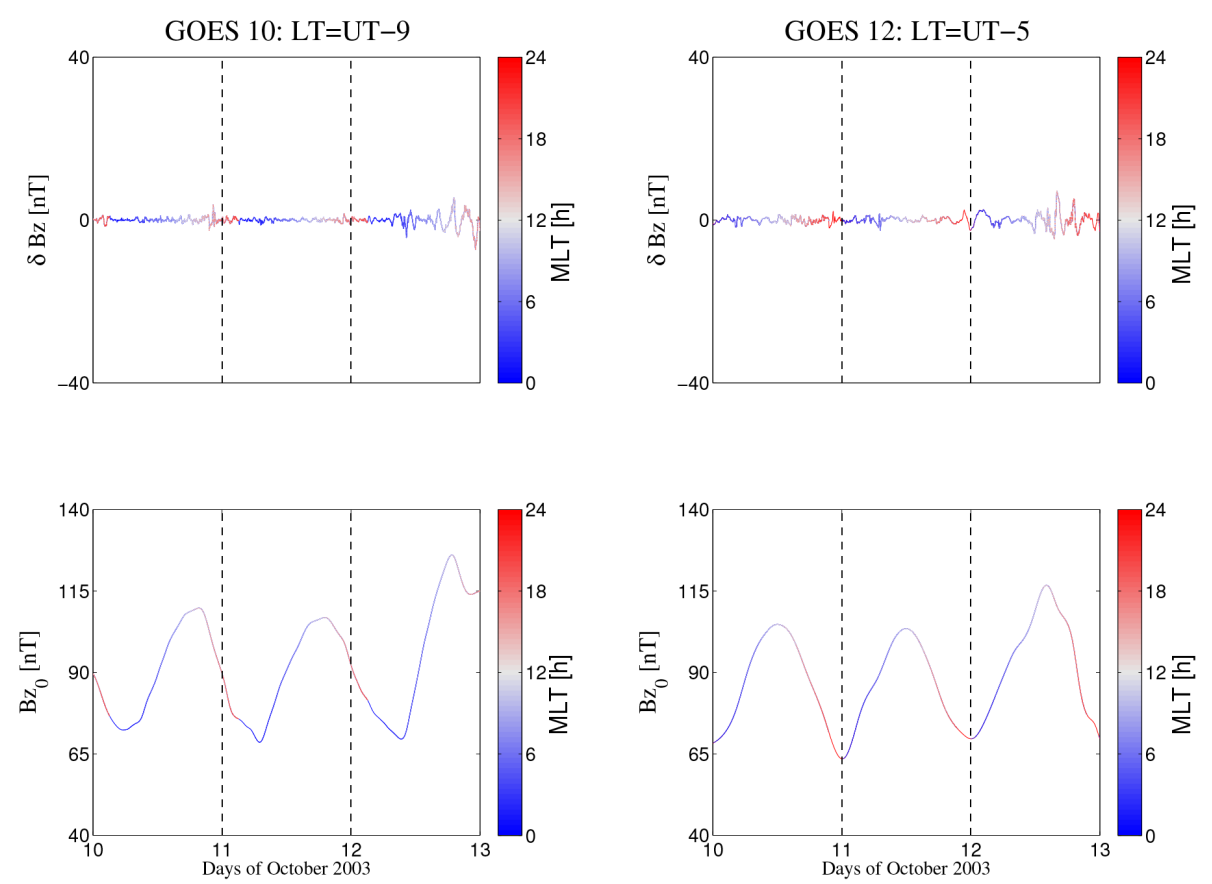

Figure 8. SSQ, 10-12 October 2003: EMD reconstruction for GOES10 (left panels) and GOES12 (right panels) geosynchronous observations. The time resolution is $1 \mathrm{~min}$.

(GOES10: $j=7-9$ modes and $r(t)$; GOES12: $j=8-9$ modes and $r(t))$.

\subsection{Storm time event: 28 October-1 November 2003}

On the basis of the result described above, we applied the same approach to the Halloween super storm (28 October-1 November 2003).

Figure 9 shows the observations at AQU geomagnetic observatory for the north-south ( $H$, left panel) and the eastwest ( $D$, right panel) components during this time interval. The solar activity at the end of October 2003 produced intense magnetospheric disturbances during three successive deep reductions in the Dst index (not shown) due to two consecutive coronal mass ejections (CMEs) which impacted the
Earth's magnetosphere (Chi et al., 2005; Balasis et al., 2012; Mannucci et al., 2014; Balasis et al., 2015). The Halloween super storm, 29-31 October 2003, has received considerable interest and analysis from both ground and space instrumentation, as it offers a great opportunity of understanding the response of the magnetosphere-ionosphere system to strong and continuous driving. At midlatitudes, the $H$ component shows a first storm peak $(\sim-500 \mathrm{nT}$ at 06:58 UT on 29 October) with an associated short recovery phase, a second storm peak ( $\sim-250 \mathrm{nT}$ at 20:13 UT on 29 October) with an irregular recovery phase, and a third peak $(\sim-280 \mathrm{nT}$ at 22:53 UT on 30 October) associated with a longer recovery phase. 
AQU: $\lambda_{g}=42.38^{\circ} \mathrm{N} ; \phi_{g}=13.32^{\circ} \mathrm{E}$
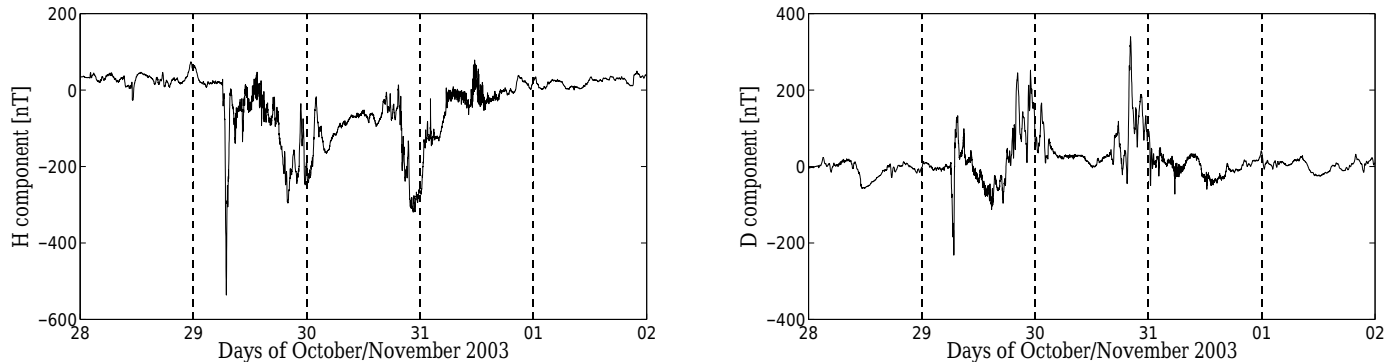

Figure 9. Halloween super storm, 28 October-1 November 2003: midlatitude ground observations (AQU: $\lambda_{\mathrm{g}}=42.38^{\circ} \mathrm{N}$ and $\left.\phi_{\mathrm{g}}=13.32^{\circ} \mathrm{E}\right)$ for the $H$ (left panel) and $D$ (right panel) components. The time resolution is $1 \mathrm{~min}$.
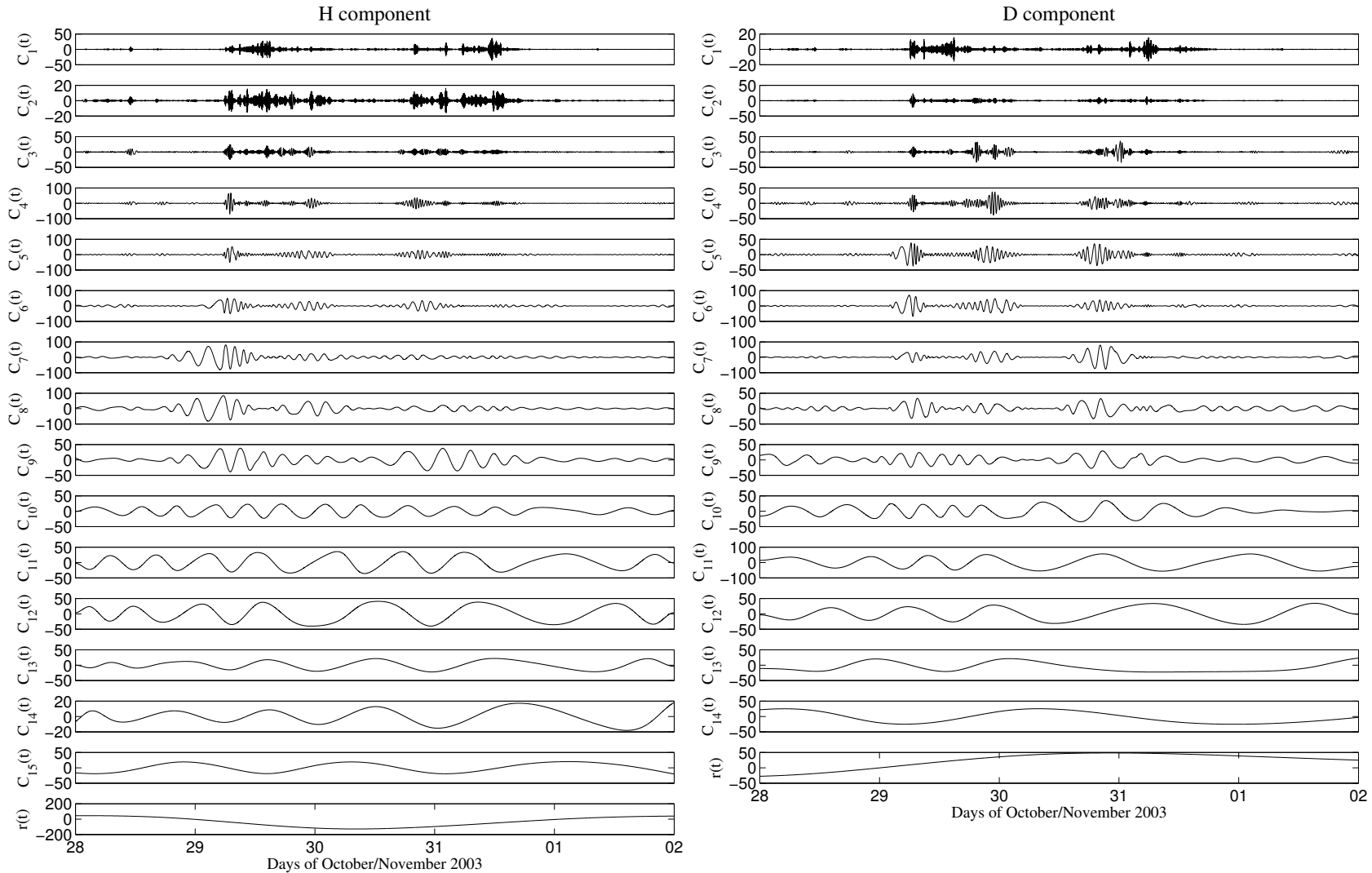

Figure 10. Halloween super storm, 28 October-1 November 2003: empirical modes extracted via EMD from $H$ (left panels) and $D$ (right panels) components recorded at AQU observatory. The modes and the residue are expressed in nanotesla, and the time resolution is 1 min

By applying the EMD procedure we found a set of 15 modes and 14 modes for the $H$ and $D$ components, respectively (see Fig. 10). We note an enhancement of the amplitude of short-timescale modes due to the arrival of the CMEs. Moreover, the characteristic diurnal contribution related to the SQ is also present $(j=14$ for $H ; j=12$ for $D)$. Figure 11 shows the SMT applied to the EMD of the $H$ (left panel) and the $D$ (right panel) components. Also for this event, we found three different sets of modes which we re- constructed as shown in Fig. 12 ( $H$ (left panels) and $D$ (right panels) components). According to the SMT results, AQU data are split into three different contributions: $\delta H(t)$ and $\delta D(t)$ (Fig. 12, upper panels) with timescales $\leq 4 \mathrm{~h}, H^{*}(t)$ and $D^{*}(t)$ (Fig. 12, middle panels) with timescales in the range of 6-24 h, and $H_{0}(t)$ and $D_{0}(t)$ (Fig. 12, lower panels) with timescales greater than $24 \mathrm{~h}$.

As for the SSQ case, we analyzed the magnetospheric observations which are shown in Fig. 13 by using a color scale 
SMT results

[AQU: $\left.\lambda_{g}=42.38^{\circ} \mathrm{N} ; \phi_{g}=13.32^{\circ} \mathrm{E}\right]$
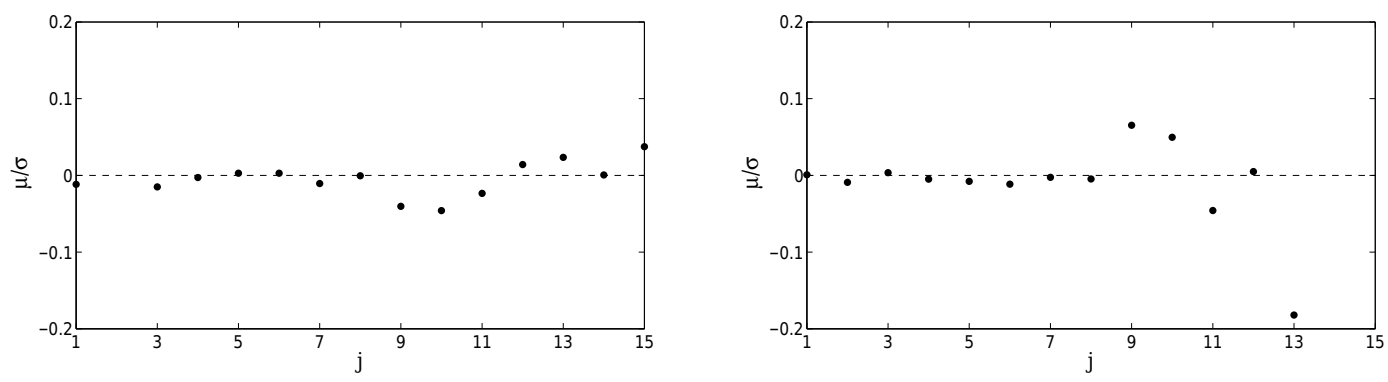

Figure 11. Halloween super storm, 28 October-1 November 2003: SMT applied to the EMD reconstruction of the ground observations for the $H$ (left panel) and $D$ (right panel) components. The $x$ axis is order in terms of the last mode involved in the partial sums (see Eq. 8).
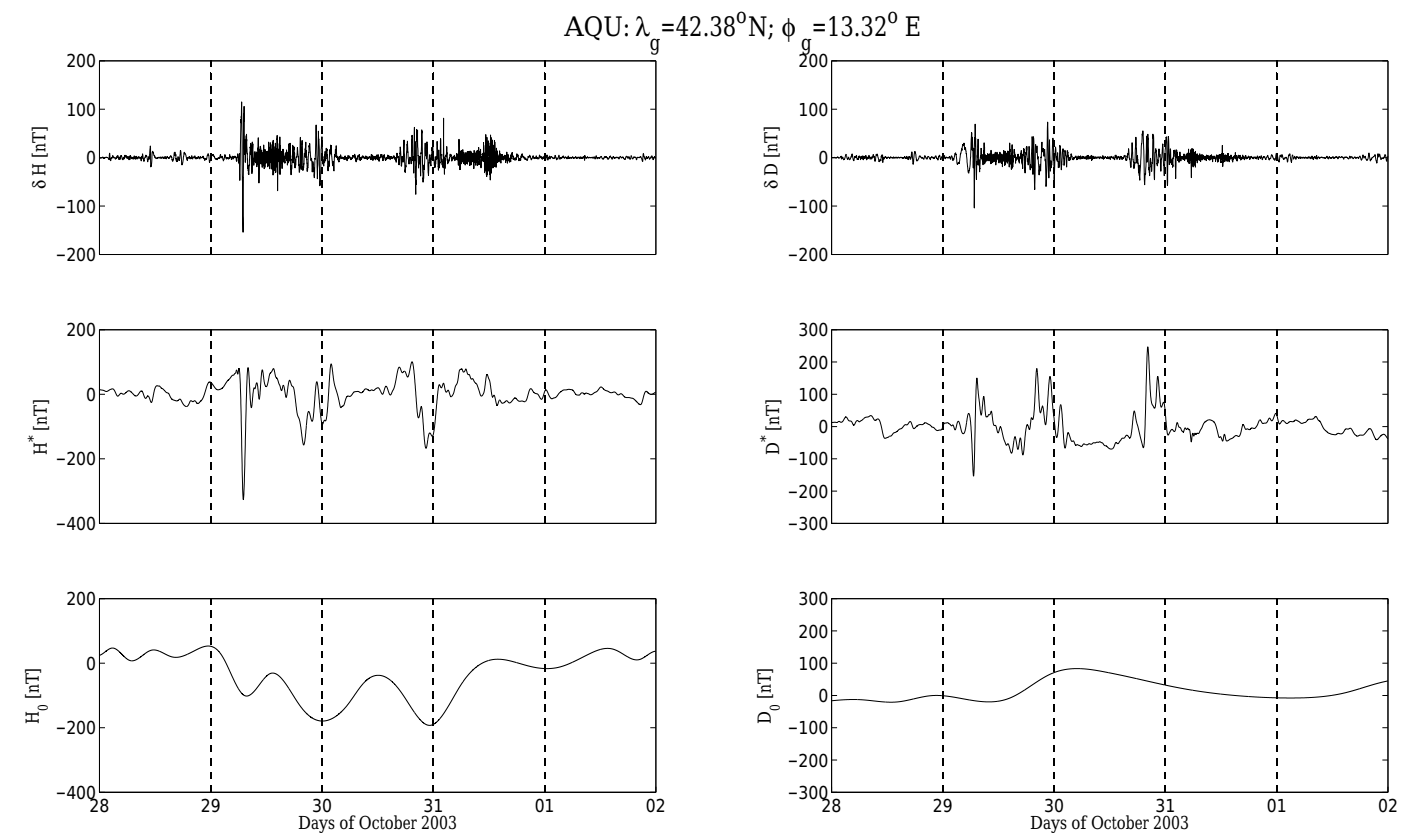

Figure 12. Halloween super storm, 28 October-1 November 2003: EMD reconstruction for the $H$ (left panels) and $D$ (right panels) components at midlatitude ground station (AQU: $\lambda_{\mathrm{g}}=42.38^{\circ} \mathrm{N}$ and $\phi_{\mathrm{g}}=13.32^{\circ} \mathrm{E}$ ). The time resolution is $1 \mathrm{~min}$.

for the MLT. In this case, a superposition of current systems can be seen at noon, related to the magnetopause current, and at midnight, due to tail current contribution.

Figure 14 shows the empirical modes extracted from GOES observations during the Halloween storm. In this case, we also note the enhancements of amplitudes due to the impact of the CMEs and their effects on the orbital variation (see $j=12-13$ for GOES10, $j=11-12$ for GOES12). At a geosynchronous orbit (Fig. 15), the SMT analysis shows only two different sets of modes for both GOES10 (left panels) and GOES12 (right panels). As a consequence, GOES10 and GOES12 are split into two different contributions: $\delta B_{z}(t)$ (GOES10: $j=1-10$ modes; GOES12: $j=1-$ 9 modes) and $B_{z 0}(t)$ (GOES10: $j=11-15$ modes and $r(t)$;
GOES12: $j=10-14$ modes and $r(t))$. Figure 16 shows the results of the EMD reconstructions for GOES10 (left panels) and GOES12 (right panels). As expected, $\delta B_{z}(t)$ in both GOES spacecraft orbits (Fig. 16, upper panels) increases during the main phase of the geomagnetic storm as a consequence of the magnetospheric response to the CME arrival and comes back close to its initial values at the end of the storm time. Concerning the GOES baseline $B_{z 0}(t)$ (Fig. 16, lower panels), it is evident that

- before the storm it is characterized by the typical diurnal variation

- during the storm it is characterized by a huge decrease due to the increase in the intensities of the magneto- 

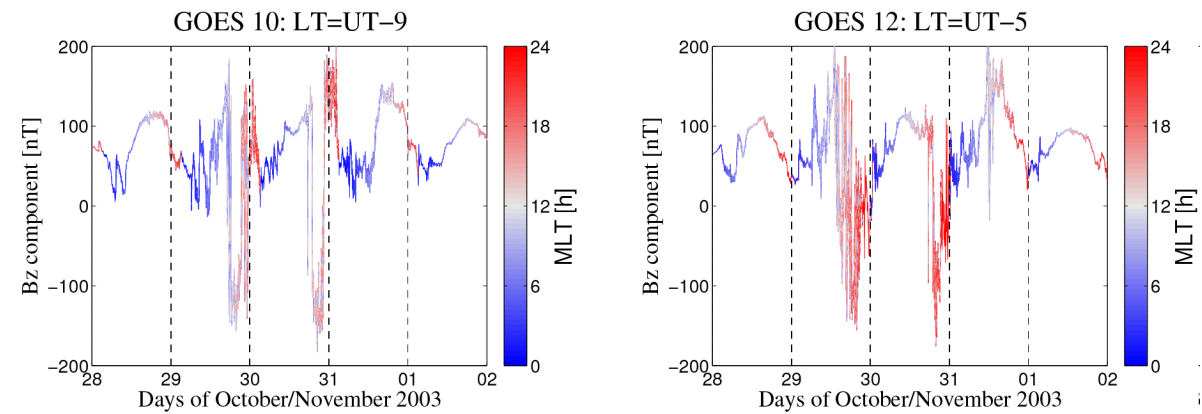

Figure 13. Halloween super storm, 28 October-1 November 2003: magnetospheric observations of the $B_{z}$ component in geosynchronous orbit for GOES10 (LT = UT-9; left panel) and GOES12 (LT=UT-5; right panel) spacecraft. The time resolution is 1 min.
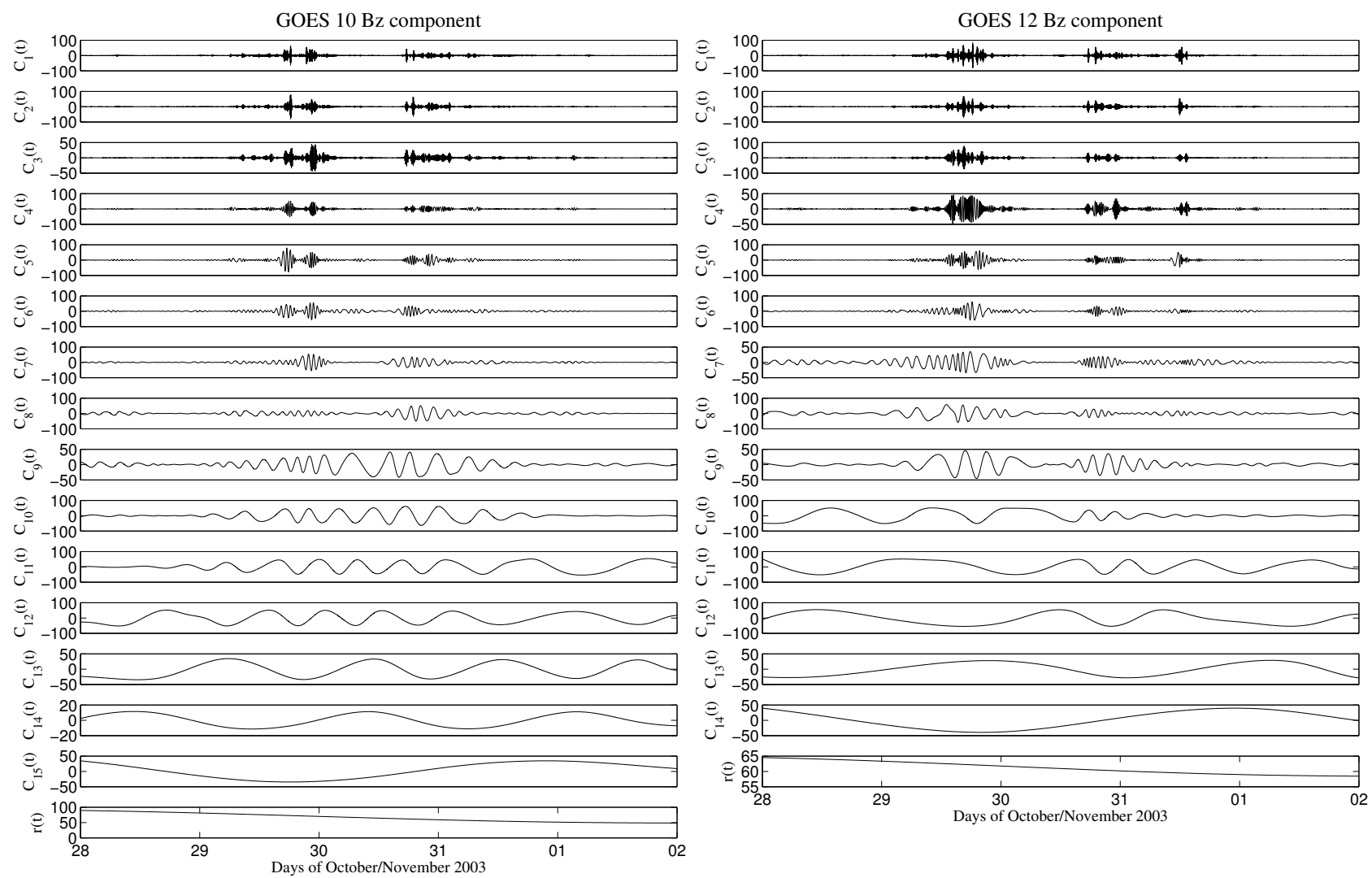

Figure 14. Halloween super storm, 28 October-1 November 2003: empirical modes extracted via EMD from GOES10 (left panels) and GOES12 (right panels) $B_{z}$ components. The modes and the residues are expressed in nanotesla, and the time resolution is 1 min.

spheric currents (the main phase of the geomagnetic storm)

- after the storm it comes back to its original diurnal variation.

Moreover, $B_{z 0}(t)$ behavior is influenced by the ring and magnetopause current activity at noon, while at midnight the superimposed effect of the ring and tail currents can be seen, with a greater contribution related to the tail current activity.
In Table 3 we report the percentage of each contribution to the total signal for both SSQ and ST periods. During the SSQ period the ground signal ( $H$ and $D$ components) variations are principally due to $H^{*}(t)$ and $D^{*}(t)(\sim 95 \%)$, while for geostationary observations they are mostly reproduced by using the baseline $B_{z 0}(t)(\sim 99 \%)$. Conversely, during the ST period, the short-timescale reconstructions $(\delta H(t)$ and $\delta D(t)$ for ground measurements and $\delta B_{z}(t)$ for magnetospheric observations) contribute more significantly to the signals $(\sim 30 \%)$. 


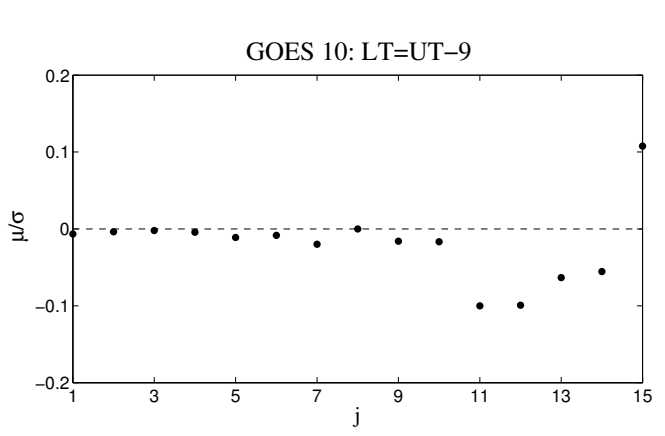

SMT results

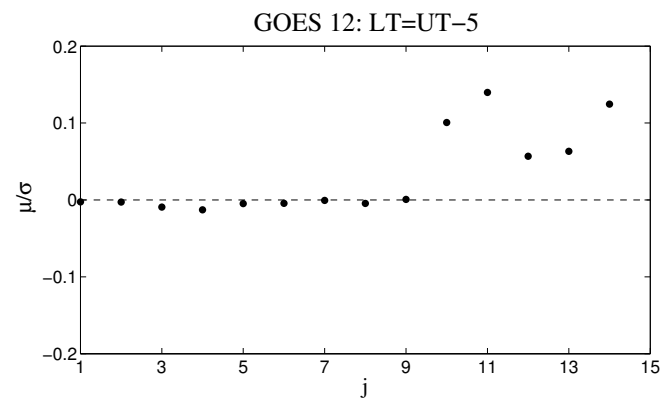

Figure 15. Halloween super storm, 28 October-1 November 2003: SMT applied to the EMD reconstruction of the magnetospheric observations for GOES10 (left panel) and GOES12 (right panel) $B_{z}$ components. The $x$ axis is order in terms of the last mode involved in the partial sums (see Eq. 8).
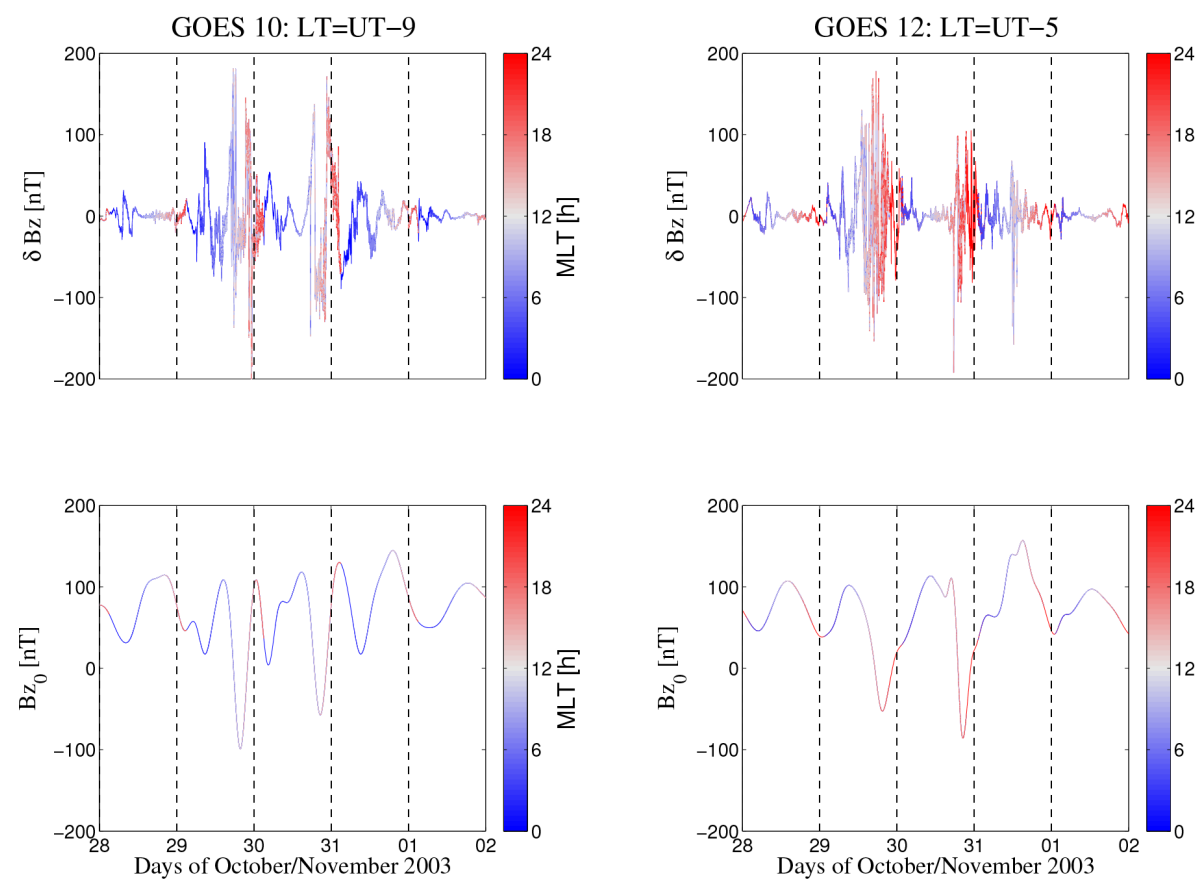

Figure 16. Halloween super storm, 28 October-1 November 2003: EMD reconstruction for GOES10 (left panels) and GOES12 (right panels) geosynchronous observations. The time resolution is $1 \mathrm{~min}$.

\section{Results and discussion}

In this work we applied the EMD to satellite and groundbased observations of the Earth's magnetic field during both quiet and disturbed periods in order to detect a timescale separation between the baseline and the time variations of the magnetospheric and ground magnetic field.

\subsection{SSQ contributions}

In ground-based observations we found three different contributions: the short-timescale contribution that can be associated with the internal dynamics of the magnetosphere, the intermediate-timescale contribution which can be related to ionospheric processes, and the large-timescale contribution related to the local time-dependent geomagnetic field. The associations of intermediate-timescale $\left(H^{*}(t)\right.$ and $\left.D^{*}(t)\right)$ contributions with ionospheric physical processes has been made by looking at their characteristic timescales (Table 1) (Feldstein and Zeitev, 1968; Dominici et al., 1997; Fytterer et al., 2013) and taking into account that the time behavior of $H^{*}(t)$ and $D^{*}(t)$ is in agreement with the solar quiet daily variation observed in October at L'Aquila, as determined by De Michelis et al. (2010). So, our findings seem to suggest that the $H^{*}(t)$ and the $D^{*}(t)$ fields are of ionospheric origin.

This hypothesis is confirmed by analyzing the magnetospheric observations from which only two contributions are 
Table 2. Mean periods of intrinsic modes during the Halloween storm (the dash corresponds to the period of the residue $r(t)$ which cannot be evaluated).

\begin{tabular}{|c|c|c|c|c|}
\hline \multicolumn{5}{|c|}{ Halloween super storm periods (h) } \\
\hline & $T_{H}$ & $T_{D}$ & $T_{G 10}$ & $T_{G 12}$ \\
\hline \multirow{10}{*}{$\delta H(D) / \delta B_{z}$} & $0.06 \pm 0.01$ & $0.06 \pm 0.01$ & $0.06 \pm 0.02$ & $0.06 \pm 0.01$ \\
\hline & $0.12 \pm 0.03$ & $0.12 \pm 0.02$ & $0.11 \pm 0.03$ & $0.11 \pm 0.02$ \\
\hline & $0.23 \pm 0.04$ & $0.22 \pm 0.02$ & $0.19 \pm 0.03$ & $0.29 \pm 0.03$ \\
\hline & $0.41 \pm 0.07$ & $0.45 \pm 0.03$ & $0.32 \pm 0.05$ & $0.49 \pm 0.04$ \\
\hline & $0.75 \pm 0.08$ & $0.71 \pm 0.06$ & $0.57 \pm 0.05$ & $0.87 \pm 0.05$ \\
\hline & $1.3 \pm 0.2$ & $1.1 \pm 0.2$ & $0.96 \pm 0.08$ & $1.3 \pm 0.2$ \\
\hline & $2.4 \pm 0.2$ & $1.8 \pm 0.3$ & $1.4 \pm 0.2$ & $2.2 \pm 0.3$ \\
\hline & $3.4 \pm 0.5$ & $2.8 \pm 0.5$ & $2.3 \pm 0.3$ & $2.9 \pm 0.5$ \\
\hline & & & $3.0 \pm 0.4$ & $3.7 \pm 0.6$ \\
\hline & & & $3.9 \pm 0.4$ & \\
\hline \multirow{4}{*}{$H^{*}\left(D^{*}\right)$} & $5.6 \pm 0.6$ & $5.8 \pm 0.6$ & & \\
\hline & $8.6 \pm 0.7$ & $12 \pm 1$ & & \\
\hline & $12 \pm 1$ & $16 \pm 1$ & & \\
\hline & $24 \pm 1$ & $24 \pm 1$ & & \\
\hline \multirow{6}{*}{$H_{0}\left(D_{0}\right) / B_{z 0}$} & $30 \pm 2$ & $43 \pm 4$ & $18 \pm 1$ & $20 \pm 1$ \\
\hline & $38 \pm 3$ & - & $24 \pm 1$ & $24 \pm 1$ \\
\hline & $48 \pm 6$ & & $34 \pm 3$ & $33 \pm 2$ \\
\hline & - & & $41 \pm 3$ & $38 \pm 3$ \\
\hline & & & $48 \pm 4$ & $47 \pm 5$ \\
\hline & & & - & - \\
\hline
\end{tabular}

Table 3. Percentage of each contribution to the total signal (\%) during both SSQ and ST periods.

\begin{tabular}{lrr}
\hline \multicolumn{3}{c}{$\begin{array}{l}\text { Percentage of each contribution } \\
\text { to the total signal }(\%)\end{array}$} \\
\hline Contribution & SSQ & ST \\
\hline$\delta B_{z}$ & 1 & 28 \\
$B_{z 0}$ & 99 & 72 \\
\hline$\delta H$ & 2 & 33 \\
$H^{*}$ & 95 & 62 \\
$H_{0}$ & 3 & 5 \\
\hline$\delta D$ & 1 & 28 \\
$D^{*}$ & 97 & 63 \\
$D_{0}$ & 2 & 9 \\
\hline
\end{tabular}

detected. It can easily be seen (Fig. 8, lower panels) that $B_{z 0}(t)$ represents the diurnal magnetospheric field variation due to the geosynchronous orbit (Rufenach et al., 1992), while $\delta B_{z}(t)$ (Fig. 8, upper panels) is related to its fluctuations ( 1 order of magnitude lower than $B_{z 0}(t)$ ). Interestingly, since $B_{z 0}(t)$ by definition represents the baseline field observed by GOES and it is completely free of any magnetospheric field fluctuations, it can be used for the calibration of the International Geomagnetic Reference Field/Definitive Geomagnetic Reference Field (IGRF/DGRF). In fact, by a direct comparison between an IGRF model, such as the Geomag 7.0C (Thebault et al, 2015), and $B_{z 0}(t)$, the IGRF degree coefficients can be tuned and better evaluated.

Since the only difference between ground and magnetospheric data during an SSQ period is the ionospheric contribution, we can reasonably assert that $H^{*}(t)$ and $D^{*}(t)$ are of ionospheric origin. Moreover, the characteristic periods of the modes involved in the $\delta H(t)$ and $\delta D(t)$ reconstructions are consistent with the relative characteristic periods involved in the $\delta B_{z}(t)$ reconstruction for both GOES spacecraft (Table 1). This indicates that they might be of magnetospheric origin.

\subsection{ST contributions}

As for the SSQ case, both $H$ and $D$ components can be split into three different contributions. Both $\delta H(t)$ and $\delta D(t)$ (Fig. 12, upper panels) show two increases in the fluctuation amplitudes as a consequence of the magnetospheric response to two consecutive CME impacts on the Earth's magnetosphere (Chi et al., 2005; Balasis et al., 2012; Mannucci et al., 2014). $H_{0}(t)$ (Fig. 12 lower left panel) gives a representation of the geomagnetic disturbance associated with the Halloween super storm (Balasis et al., 2012), characterized by three storm peaks and the relative recovery phases, while $D_{0}(t)$ (Fig. 12, lower right panel) shows only a slight modulation around $0 . H^{*}(t)$ and $D^{*}(t)$ (Fig. 12, middle panels), which are of ionospheric origin, show variations between 
6-24h which increase in amplitude during the storm time, with amplitude peaks of $\sim-250$ and $\sim-200 \mathrm{nT}$, respectively, during the first main phase. Moreover, $D^{*}(t)$ shows two huge increases $(\sim 220$ and $\sim 270 \mathrm{nT})$ during the second and the third main phase of the storm.

\section{Summary}

Our results can be summarized as reported below.

\subsection{Magnetosphere}

Both in the quiet (Fig. 5) and storm time periods (Fig. 13), the SMT applied to the EMD shows two different contributions:

1. Short-timescale $\delta B_{z}(t)$ : this gives a representation of the magnetospheric field variations probably due to the dynamic of the ring current system. In fact, in the SSQ (Fig. 8, upper panels) and in the GS (Fig. 16, upper panels) periods, both GOES spacecraft orbit in the ring current (De Michelis et al., 1999).

2. Baseline $B_{z 0}(t)$ : this describes the magnetospheric field observed by the spacecraft during its diurnal orbit (Fig. 8, lower panels). It has a periodicity of $24 \mathrm{~h}$ and an average value of $\sim 90 \mathrm{nT}$ at $6.6 R_{\mathrm{E}}$ (http://www.ngdc. noaa.gov/IAGA/vmod/igrfhw.html), during both SSQ and the pre-storm period. For this reason, during SSQ conditions, it could be used for a local calibration of the IGRF model in the magnetosphere. Moreover, its time evolution can be used as a measure of the local magnetospheric current activity, especially during storm time periods when fluctuations increase and the evaluation of an average magnetic field is not straightforward (Fig. 16, lower panels). In fact, $B_{z 0}(t)$ shows large variations during the different phases of the storm and a smooth recovery to its diurnal variation after the storm.

\subsection{Ground}

Both in the SSQ (Fig. 1) and in the storm period (Fig. 9), the SMT applied to the EMD shows three different contributions:

1. Short-timescale $\delta H(t)$ and $\delta D(t)$ : they represent the geomagnetic field variations due to the magnetospheric field fluctuations. The similar temporal scales of $\delta H(t)$ and $\delta B_{z}(t)$ (Table 1 and Table 2) seem to confirm this hypothesis. A visual inspection of the interplanetary magnetic field ( $B_{z}$, IMF, not shown) seems to suggest that any coherent increase (decrease) in $\delta H(t)$ is correlated with the northward (southward) switching of the $B_{z, \mathrm{IMF}}$

2. Intermediate-timescale $H^{*}(t)$ and $D^{*}(t)$ : they represent the geomagnetic field variations of ionospheric origin, as confirmed by their absence in the magnetospheric EMD reconstructions and by their characteristic timescales (Table 1 and Table 2). In both SSQ and storm conditions, both $H^{*}(t)$ and $D^{*}(t)$ show periods in the range of 6-24 $\mathrm{h}$ (Fig. 4, middle panels). In addition, during a GS, $H^{*}(t)$ increases in amplitude, with higher values in the main phase. By contrast, $D^{*}(t)$ shows a decrease in the first main phase and two huge increases of $\sim 220$ and $\sim 270 \mathrm{nT}$ in the second and in the third main phase of the storm (Fig. 12, middle panels), respectively.

3. Baseline $H_{0}(t)$ and $D_{0}(t)$ : they represent the local average deviation of the geomagnetic field. During the SSQ (Fig. 4, lower panels), their mean value is set around 0 , as a consequence of the lack of a baseline value from AQU ground measurements (we used the relative measurements of the geomagnetic field variations recorded by a triaxial fluxgate magnetometer). On the other hand, during the storm time period (Fig. 12, lower panels), $H_{0}(t)$ shows the typical behavior of a geomagnetic storm, characterized by huge decreases (main phases) followed by a smooth increase in the magnetic field (recovery phases).

\section{Conclusions}

We provide a method to easily discriminate between the average magnetic field and its time variations in both magnetospheric and ground observations. The SMT applied to the EMD is capable of identifying the physical meaning of each evaluated contribution. In the magnetosphere, we associated the large-timescale variation (baseline) to the magnetic field observed by the spacecraft during its diurnal orbit (Rufenach et al., 1992). During a GS, this baseline shows large amplitude variations, and it could be used as a measure of the local magnetospheric current activity by a comparison between $B_{z 0}(t)$ and the TS04 magnetospheric field model (Tsyganenko and Sitnov, 2005). In fact, since the TS04 model is modular, it can be used to evaluate the magnetospheric current that best fits the $B_{z 0}(t)$ variations (Villante and Piersanti, 2008; Piersanti and Villante, 2016).

Moreover, during an SSQ period, the same baseline could be used to efficiently calibrate the IGRF model. On the other hand, the short-timescale variations could be related to the magnetospheric field fluctuations that can tentatively be associated with the symmetric and partial ring current dynamics (De Michelis et al., 1999). On the ground, we associated the short-timescale reconstructions with the variations of the geomagnetic field driven by the different magnetospheric current system dynamics. This is confirmed by the similar timescales between $\delta H(t)$ and $\delta B_{z}(t)$ (Tables 1 and 2). In addition, we found intermediate-timescale variations that we associated with an ionospheric origin contribution. This is confirmed by their absence in the magnetospheric reconstructions and by their characteristic time periods in the range of 6-24 h. Moreover, we connected the large-timescale variations to the local average of the geomagnetic field. In fact, 
for SSQ, the local average shows a mean value set around 0, while during active magnetic conditions, the baseline $\left(H_{0}(t)\right)$ presents the typical GS feature, characterized by sudden impulse, main phase, and recovery. Thus, $H_{0}(t)$ might be used for the evaluation of the local intensity of a GS on the ground because it is not necessary to calculate and subtract any average value for its evaluation (including the SQ field) and it is free of any magnetospheric fluctuations and ionospheric origin contributions. Since it is easily evaluated at any ground and magnetospheric observatory, it can be used as a local geomagnetic disturbance index.

Interestingly, on the ground we explicitly separated the ionospheric and magnetospheric fluctuations from the observations. This could be very useful in evaluating the local ionospheric and magnetospheric responses to the solar wind conditions. Further investigation on several other storm events are in progress.

\section{Data availability}

The GOES magnetic field data were provided by H. Singer (National Oceanic and Atmospheric Administration Space Environment Center, Asheville, NC, USA) through the NASA's cdaweb site (http://cdaweb.gsfc.nasa.gov.) (Singer, 2016). The results presented in this paper rely on public data collected at AQU permanent geomagnetic observatory which are available through INTERMAGNET (2016) (www.intermagnet.org). The full data for this paper are available by contacting the corresponding author.

Acknowledgements. The authors thank the reviewer for his/her useful comments and suggestions and G. Consolini (Istituto di Astrofisica e Planetologia Spaziali) for his helpful cooperation. We thank the INGV that supports INTERMAGNET to promote high standards of magnetic observatory practice. This work is supported by the Italian Ministry for Education and Research MIUR PRIN Grant No. 2012P2HRCR on "The active Sun and its effects on Space and Earth climate".

The topical editor, G. Balasis, thanks two anonymous referees for help in evaluating this paper.

\section{References}

Alberti, T., Lepreti, F., Vecchio, A., Bevacqua, E., Capparelli, V., and Carbone, V.: Natural periodicities and Northern HemisphereSouthern Hemisphere connection of fast temperature changes during the last glacial period: EPICA and NGRIP revisited, Clim. Past, 10, 1751-1762, doi:10.5194/cp-10-1751-2014, 2014.

Balasis G. and Egbert G. D.: Empirical orthogonal function analysis of magnetic observatory data: Further evidence for non-axisymmetric magnetospheric sources for satellite induction studies, Geophys. Res. Lett., 33, L11311, doi:10.1029/2006GL025721, 2006.

Balasis, G., Daglis, I. A., Zesta, E., Papadimitriou, C., Georgiou, M., Haagmans, R., and Tsinganos, K.: ULF wave activity during the 2003 Halloween superstorm: multipoint observations from CHAMP, Cluster and Geotail missions, Ann. Geophys., 30, 1751-1768, doi:10.5194/angeo-30-1751-2012, 2012.

Balasis, G., Daglis, I. A., Mann, I. R., Papadimitriou, C., Zesta, E., Georgiou, M., Haagmans, R., and Tsinganos, K.: Multi-satellite study of the excitation of Pc3 and Pc4-5 ULF waves and their penetration across the plasmapause during the 2003 Halloween superstorm, Ann. Geophys., 33, 1237-1252, doi:10.5194/angeo33-1237-2015, 2015.

Chapman, S.: On the theory of the solar diurnal variation of the Earth's magnetism, Proc. R. Soc. Lon. Ser. A, 129, 369-386, 1929.

Chi, P. J., Russell, C. T., Foster, J. C., Moldwin, M. B., Engebretson, M. J., and Mann, I. R.: Density enhancement in plasmasphereionosphere plasma during the 2003 Halloween Superstorm: Observations along the 330th magnetic meridian in North America, Geophys. Res. Lett., 32, L03S07, doi:10.1029/2004GL021722, 2005.

Chulliat, A., Blanter, E., Le Mouel, J. L., and Shnirman, M.: On the seasonal asymmetry of the diurnal and semidiurnal geomagnetic variations, J. Geophys. Res., 110, A05301, doi:10.1029/2004JA010551, 2005.

Cid, C., Palacios, J., Saiz, E., Guerrero, A., and Cerrato, Y.: On extreme geomagnetic storms, J. Space Weather Space Clim., 4, A28, doi:10.1051/swsc/2014026, 2014.

Cummings, D. A. T., Irizarry, R. A., Huang, N. E., Endy, T. P., Nisalak, A., Ungchusak, K., and Burke, D. S.: Travelling waves in the occurrence of dengue haemorrhagic fever in Thailand, Nature, 427, 344-347, doi:10.1038/nature02225, 2004.

De Michelis, P., Daglis, I. A., and Consolini, G.: An average image of proton plasma pressure and of current systems in the equatorial plane derived from AMPTE/CCE-CHEM measurements, J. Geophys. Res., 104 (A12), 28615-28624, doi:10.1029/1999JA900310, 1999.

De Michelis, P., Tozzi, R., and Consolini, G.: Principal components' features of mid-latitude geomagnetic daily variation, Ann. Geophys., 28, 2213-2226, doi:10.5194/angeo-28-2213-2010, 2010.

De Michelis, P., Consolini, G., and Tozzi, R.: On the multi-scale nature of large geomagnetic storms: an empirical mode decomposition analysis, Nonlin. Processes Geophys., 19, 667-673, doi:10.5194/npg-19-667-2012, 2012.

Dessler, A. J. and Parker, E. N.: Hydromagnetic theory of magnetic storms, J. Geophys. Res., 64, 2239-2259, 1959.

Dominici, P., Cander, L. R., and Zolesi, B.: On the origin of medium-period ionospheric waves and their possible modelling: a short review, Annals of Geophysics, [S.1.], 40, n. 5, ISSN $2037-$ 416X, 1997.

Feldstein, Y. I. and Zaitzev, A. N., Quiet and disturbed solar-daily variations of magnetic field at high latitudes during the IGY, Tellus, 20, 338-366. doi:10.1111/j.2153-3490.1968.tb00376.x, 1968.

Fytterer, T., Arras, C., and Jacobi, C.: Terdiurnal signatures in sporadic $E$ layers at midlatitudes, Adv. Radio Sci., 11, 333-339, doi:10.5194/ars-11-333-2013, 2013.

Flandrin, P., Gonçalvès, P., and Rilling, G.: Detrending and denoising with empirical mode decompositions, Proceedings of Eusipco, Wien, Austria, 2004.

Gonzáles-Hernámdez, I., Komm, R., Pevtsov, A., and Leibacher, J.: Solar Origin of Space Weather and Space Climate, Springer, New York, USA, ISBN 978-1-4939-1181-3, 2014. 
Goodman, J. M.: Space Weather \& Telecommunications, Springer, New York, USA, ISBN 0-387-23670-8, 2005.

Hawary, R. E., Yumoto, K., Yamazaki, Y., Mahrous, A., Ghamry, E., Meloni, A., Badi, K., Kianji, G., Uiso, C. B. S., Mwiinga, N., Joao, L., Affluo, T., Sutcliffe, P. R., Mengistu, G., Baki, P., Abe, S., Ikeda, A., Fujimoto, A., and Tokunaga, T: Annual and semi-annual $\mathrm{Sq}$ variations at $96^{\circ}$ MM MAGDAS I and II stations in Africa, Earth Planets Space, 64, 425-432, doi:10.5047/eps.2011.10.013, 2012.

Huang, N. E. and Wu, Z.: A review on Hilbert-Huang transform: Method and its applications to geophysical studies, Rev. Geophys., 46, RG2006, doi:10.1029/2007RG000228, 2008.

Huang, N. E., Shen, Z., Long, S. R., Wu, M. C., Shih, H. H., Zheng, Q., Yen, N., Tung, C. C., and Liu, H. H.: The empirical mode decomposition and the Hilbert spectrum for nonlinear and nonstationary time series analysis, Proc. R. Soc. Lon. Ser. A, 454, 903-995, 1998.

Hughes, W. J. and Southwood, D. J.: Effect of the atmosphere and ionosphere on magnetospheric micropulsation signals, Nature, 248, 493-495, doi:10.1038/248493b0, 1974.

INTERMAGNET: AQU magnetic observatory data, available at: www.intermagnet.org, last access: 11 November 2016.

Jánosi, I. M. and Müller, R.: Empirical mode decomposition and correlation properties of long daily ozone records, Phys. Rev. E, 71, 1597-1611, 2005.

Mannucci, A. J., Crowley, G., Tsurutani, B. T., Verkhoglyadova, O. P., Komjathy, A., and Stephens, P.: Interplanetary magnetic field By control of prompt total electron content increases during superstorms, J. Atmos. Sol.-Terr. Phy., 115, 7-16, doi:10.1016/j.jastp.2014.01.001, 2014.

Matsushita, S. and Maeda, H.: On the geomagnetic quiet daily variation field during the IGY, J. Geophys. Res., 70, 2535-2558, 1965.

McDonald, A. J., Baumgaertner, A. J. G., Fraser, G. J., George, S. E., and Marsh, S.: Empirical Mode Decomposition of the atmospheric wave field, Ann. Geophys., 25, 375-384, doi:10.5194/angeo-25-375-2007, 2007.

McPherron, R. L., Baker, D. N., and Bargatze, L. F.: Linear filters as a method of real time prediction of geomagnetic activity, in: Solar Wind Magnetosphere Coupling, edited by: Kamide, Y. and Slavin, J. A., 85-92, 1986.

Piersanti, M. and Villante, U.: On the discrimination between magnetospheric and ionospheric contributions on the ground manifestation of sudden impulses, J. Geophys. Res.-Space, 121, 6674-6691, doi:10.1002/2015JA021666, 2016.

Piersanti, M., Villante, U., Waters, C., and Coco, I.: The 8 June 2000 ULF wave activity: A case study, J. Geophys. Res., 117, A02204, doi:10.1029/2011JA016857, 2012.

Pulkkinen, T.: Space Weather: Terrestrial Perspective, Living Rev. Solar Phys., 4, 1, doi:10.12942/lrsp-2007-1, 2007.

Richmond, A. D., Matsushita, S., and Tarpley, J. D.: On the production mechanism of electric currents and fields in the ionosphere. J. Geophys. Res., 81, 547-555, doi:10.1029/JA081i004p00547, 1976.

Rufenach, C. L., McPherron, R. L., and Schaper, J.: The quiet geomagnetic field at geosynchronous orbit and its dependence on solar wind dynamic pressure, J. Geophys. Res., 97, 25-42, doi:10.1029/91JA02135, 1992

Schwenn, R.: Space Weather: The solar perspective, Living Rev. Solar Phys., 3, 2, doi:10.12942/lrsp-2006-2, 2006.
Sciffer, M. D., Waters, C. L., and Menk, F. W.: Propagation of ULF waves through the ionosphere: Inductive effect for oblique magnetic fields, Ann. Geophys., 22, 1155-1169, doi:10.5194/angeo22-1155-2004, 2004.

Sckopke, N.: A general relation between the energy of trapped particles and the disturbance field near the Earth, J. Geophys. Res., 71, 3125-3130, 1966.

Shinbori A., Koyama, Y., Nose, M., Hori, T., Otsuka, Y., and Yatagai, A.: Long-term variation in the upper atmosphere as seen in the geomagnetic solar quiet daily variation, Earth Planets Space, 66, 155-174, doi:10.1186/s40623-014-0155-1, 2014.

Singer, H.: The GOES magnetic field data, available at: http:// cdaweb.gsfc.nasa.gov, last access: 11 November 2016.

Takeda, M.: Features of global geomagnetic Sq field from 1980 to 1990, J. Geophys. Res., 107, 1252, doi:10.1029/2001JA009210, 2002.

Terradas, J., Oliver, R., and Ballester, J. L.: Application of Statistical Techniques to the Analysis of Solar Coronal Oscillations, Astrophys. J., 614, 435-447, doi:10.1086/423332, 2004.

Thebault, E., Finlay, C. C., and Toh, H.: International Geomagnetic Reference Field - the twelfth generation, Earth Planets Space, 67, doi:10.1186/s40623-015-0313-0, 2015.

Tsyganenko, N. A. and Sitnov, M. I.: Modeling the dynamics of the inner magnetosphere during strong geomagnetic storms, J. Geophys. Res., 110, A03208, doi:10.1029/2004JA010798, 2005.

Vecchio, A., Laurenza, M., Carbone, V., and Storini, M.: Quasibiennial Modulation of Solar Neutrino Flux and Solar and Galactic Cosmic Rays by Solar Cyclic Activity, Astrophys. J. Lett., 709, L1-L5, doi:10.1088/2041-8205/709/1/L1, 2010.

Villante, U. and Piersanti, M.: An analysis of sudden impulses at geosynchronous orbit, J. Geophys. Res., 113, A08213, doi:10.1029/2008JA013028, 2008.

Waters, C. L., Lysak, R. L., and Sciffer, M. D.: On the coupling of fast and shear Alfvén wave modes by the ionospheric Hall conductance, Earth Planets Space, 65, 2, doi:10.5047/eps.2012.08.002, 2013.

$\mathrm{Wu}, \mathrm{Z}$. and Huang, N. E.: A study of the characteristics of white noise using the Empirical mode decomposition method, Proc. R. Soc. Lon. Ser.-A., 460, 1597-1611, doi:10.5047/eps.2012.08.002, 2004.

Wu, Z. and Huang, N. E.: Ensemble Empirical Mode Decomposition: a Noise-Assisted Data Analysis Method, Adv. Adapt. Data Anal., 1, 1-41, 2009.

Wu, Z., Schneider, E. K., Kirtman, B. P., Sarachik, E. S., Huang, N. E., and Tucker, C. J.: The modulated annual cycle: an alternative reference frame for climate anomalies, Clim. Dynam., 31, 823, doi:10.1007/s00382-008-0437-z, 2008.

Yamazaki, Y. and Yumoto, K.: Long-term behavior of annual and semi-annual Sq variations, Earth Planets Space, 64, 417-423, doi:10.5047/eps.2011.01.014, 2012.

Yang, G., Sun, X.-B., Zhang, M., Li, X., and Liu, X.: Study on Ways to Restrain End Effect of Hilbert-Huang Transform, Journal of Computers, 25, 22-31, 2014.

Zhen-Shan, L. and Xian, S.: Multi-scale analysis of global temperature changes and trend of a drop in temperature in the next 20 years, Meteorol. Atmos. Phys., 95, 115-121, doi:10.1007/s00703-006-0199-2, 2007. 